\title{
Towards establishing a minimal nanoparticle concentration for applications involving surface enhanced spatially offset resonance Raman spectroscopy (SESORRS) in vivo
}

\section{Authors}

Fay Nicolson ${ }^{1}$, Lauren E. Jamieson ${ }^{1}$, Samuel Mabbott ${ }^{1}$, Konstantinos Plakas ${ }^{2}$, Neil C. Shand ${ }^{3}$, Michael R. Detty ${ }^{2}$, Duncan Graham ${ }^{1}$, Karen Faulds ${ }^{1 *}$

1. Department of Pure and Applied Chemistry, Technology and Innovation Centre, University of Strathclyde, 99 George Street, Glasgow G1 1RD, UK.

2. Department of Chemistry, University at Buffalo, The State University of New York, New York 14260, United States

3. DSTL, Porton Down, Salisbury, SP4 0JQ, UK.

Corresponding author:

Karen Faulds, Department of Pure and Applied Chemistry, Technology and Innovation Centre, University of Strathclyde, 99 George Street, Glasgow G1 1RD, UK.

E-mail: karen.faulds@strath.ac.uk

\begin{abstract}
Resonant chalcogenpyrylium nanotags demonstrate an exceptional surface enhanced Raman scattering (SERS) performance for use in SORS applications. Using surface enhanced spatially offset Raman spectroscopy (SESORS), nanotags modified with a chalcogenpyrylium dye were observed at concentrations as low as 1 pM through 5 $\mathrm{mm}$ of tissue. Calculated limits of detection suggest that these SERS nanotags can be detected at $104 \mathrm{fM}$ using surface enhanced spatially offset resonance Raman scattering (SESORRS) demonstrating their potential for in vivo applications.
\end{abstract}

\section{Introduction}

The ability to detect low levels of analytes through barriers in a sensitive and nondestructive manner is a challenge faced in both the security and biomedical fields. ${ }^{1}$ Raman spectroscopy provides a means to solving this challenge since it measures a unique chemical fingerprint that can distinguish between signals from the barrier and the analyte without the need to destroy the sample, however it is often limited in its ability to detect analytes at depth. ${ }^{2}$ Spatially offset Raman spectroscopy (SORS) is an emerging technique which is capable of providing spectral information on the analyte under study, even when obscuring barriers such as tissue are present. ${ }^{3}$ SORS relies on the idea that photons generated at depth undergo multiple diffuse scattering processes, and thus travel laterally upon return to the collection probe. ${ }^{2}$ Unlike conventional $180^{\circ}$ backscattering techniques, where excitation and collection typically take places at the same point, SORS makes use of a spatial offset between the point of laser excitation and the point of collection. ${ }^{2}$ By exploiting the use of a spatial offset between the point of incident light and the point of collection, it is possible to obtain Raman signal of the photons generated at depth, i.e. the analyte obscured by the barrier. ${ }^{4}$ Since first reported by Matousek et al., ${ }^{2}$ SORS has been applied to a number of applications including security, ${ }^{4-7}$ the detection of counterfeit alcohol ${ }^{8}$ and the monitoring of the quality of red blood cells. ${ }^{9}$ Perhaps more importantly, the introduction of SORS has opened up new avenues for medical applications; in particular noninvasive disease diagnostics. Several reports have explored the use of SORS for the 
assessment of bone ${ }^{10}$, allowing information on both the inorganic and organic components of bone to be ascertained, ${ }^{3}$ as well as in the non-invasive assessment of calcifications associated with breast cancer. ${ }^{11}$

Surface enhanced Raman spectroscopy (SERS) is useful for overcoming the limitations associated with conventional Raman spectroscopy. By functionalising nanoparticles (NPs) with a molecularly specific Raman reporter, greater enhancement in the inelastic scattering of the Raman reporter can be achieved. ${ }^{12}$ Surface enhanced resonance Raman spectroscopy (SERRS) makes use of a Raman reporter with an electronic transition close to that of the laser excitation wavelength and generates further enhancement in Raman signal. ${ }^{13}$ SERS has been used extensively in biomedical imaging applications and nanotags functionalised with biomolecules such as antibodies have assisted in the targeted imaging of numerous cancers in vivo including ovarian ${ }^{14}$ and esophageal. ${ }^{15}$

Surface enhanced spatially offset Raman spectroscopy (SESORS) encompasses both the SERS and SORS techniques ${ }^{16}$ and, whilst not a completely non-invasive technique as the NPs must be introduced, it does achieve considerable enhancement in Raman signal at larger, more clinically relevant depths. Since it was first reported by Stone et al., ${ }^{16}$ SERS active nanotags have been tracked through depths of up to $50 \mathrm{~mm}$ of tissue using a transmission optical approach. ${ }^{17}$ Using a backscattering geometry, SESORS has been used in glucose sensing, ${ }^{18}$ in the tracking of nanotags through $8 \mathrm{~mm}$ of bone ${ }^{19}$ and in the detection of neurotransmitters in the skull. ${ }^{20}$

Previous work in our group has reported the technique of surface enhanced spatially offset resonance Raman spectroscopy (SESORRS), which demonstrates the benefit of using a resonant Raman reporter for superior levels of depth penetration. ${ }^{21}$ In this instance, SERRS active nanotags were detected through $25 \mathrm{~mm}$ of porcine tissue and the same nanotags were taken up into ex vivo breast cancer models and detected through $15 \mathrm{~mm}$ of tissue using a handheld SORS spectrometer. ${ }^{21,22}$ Such depth penetration was achieved through the use of chalcogenpyrylium Raman reporters with a tuneable absorption maxima in the near infrared (NIR). We have also demonstrated, using both conventional Raman ${ }^{23}$ and SORS, ${ }^{21}$ that by using a Raman reporter that is in resonance with the laser excitation wavelength, it is possible to detect SERS nanotags through larger thicknesses in comparison to when non-resonant Raman reporters are used. Previous reports have shown these reporter molecules to be particularly useful at longer wavelengths including $1280 \mathrm{~nm}^{24}$ and $1550 \mathrm{~nm}^{25}$, outperforming commercially available Raman reporter molecules such as BPE.

In recent years, focus has shifted towards the use of handheld Raman instrumentation, mainly due to portability, ease of use and typically lower cost. ${ }^{26}$ Previous work in the SESORS field has typically focused on the depth penetration capabilities of the technique, i.e. the focus has been to probe through significant depth. However, few studies have investigated the minimum nanoparticle (NP) concentration that can be detected at a given tissue thickness. This is a key question that needs to be addressed if SESORS is to translate into the clinic where the number of nanoparticles found at a targeted site, for example, a tumour, may be low. As such, the work presented here explores the benefit of using resonant Raman reporters for low limits of detection of SERRS nanotags through tissue using a handheld SORS instrument. 


\section{Experimental}

\section{Materials and Methods}

All chemicals and small molecule Raman reporters were purchased from Sigma Aldrich unless otherwise stated. AuNPs with an average diameter of $78 \mathrm{~nm}$ were synthesized using a seeded method and left to stir overnight. ${ }^{27}$ Briefly, gold seeds of $26 \mathrm{~nm}$ were synthesized using the citrate reduction method. Sodium tetrachloroaurate (III) dihydrate $(681 \mu \mathrm{L}$, final concentration $0.254 \mathrm{M})$ and sodium citrate trihydrate (528 $\mu \mathrm{L}$, final concentration $0.171 \mathrm{M}$ ) were added to $5.007 \mathrm{ml}$ of $28 \mathrm{~nm}$ seeds and made up to $120 \mathrm{ml}$ with $\mathrm{dH}_{2} \mathrm{O}$. The solution was left to stir overnight. NPs were characterized using extinction spectroscopy and had an LSPR of $548 \mathrm{~nm}$.

A chalcogenpyrylium-based dye was synthesized according to previously reported methods. ${ }^{24,25,28}$ Dye 823 was prepared by dissolving the solid in anhydrous N,NDimethylformamide (DMF, 99.8\%) to produce a $1 \mathrm{mM}$ stock. Subsequent dilutions were then carried out using DMF and $\mathrm{dH}_{2} \mathrm{O}$ (50:50). Raman reporter 1,2-bis(4pyridyl)ethylene (BPE) was prepared by dissolving the solid in ethanol to produce a $10 \mathrm{mM}$ stock. Subsequent dilutions were carried out using $\mathrm{dH}_{2} \mathrm{O}$. Dye 823 was characterized using extinction spectroscopy (Agilent Cary 60 ) to determine the $\lambda$ max. BPE is a non-resonant Raman reporter.

Measurements were taken using a handheld Resolve instrument from Cobalt Light Systems (830 nm, average laser power $450 \mathrm{~mW}$ ). All measurements were carried out using a $2 \mathrm{~s}$ integration time, 5 accumulations and an $8 \mathrm{~mm}$ offset. The nose cone was fitted to use the instrument in a contact mode setting. The handheld instrument used here has a fixed exposure time. Measurements were carried out using 3 samples. Prior to dye addition, NPs were concentrated by centrifugation ( $1 \mathrm{~mL}$ aliquots, 5000 RPM, 10 mins) and resuspended in $500 \mu \mathrm{L}$ of water.

\section{Experimental set up}

Pork loin tissue was obtained from a local butcher and cut into sections (roughly 3.5 $\mathrm{cm}$ inches $\times 4 \mathrm{~cm}$ with varying thicknesses). Pork was chosen as an analogue to human samples due its ability to mimic human tissue greater than that from avian species. ${ }^{16}$ LOD experiments were performed using quartz cuvettes. $350 \mu \mathrm{L}$ of each nanotag solution was pipetted into a Suprasil quartz micro cuvette, path length $1 \mathrm{~mm}$, chamber volume $350 \mu \mathrm{L}$. Tissue samples of varying thicknesses were then placed in front of the cuvette. The nose cone was brought into contact with the tissue samples, thus ensuring there was no space between the instrument and the tissue. The set up involving the cuvette is shown in Error! Reference source not found..

Investigation of the nanotags for LODs using SESORS was carried out by adding each reporter $(3 \mathrm{uL}, 300 \mu \mathrm{M})$ to $500 \mu \mathrm{L}$ of NPs. The solution was then made up to $1 \mathrm{~mL}$ with $\mathrm{dH}_{2} \mathrm{O}$. An initial final dye concentration of $300 \mathrm{nM}$ was used for each nanotag solution, thus by keeping the dye concentration as low as possible the benefit of using a Raman 
reporter which is in resonance with the laser was exploited. Nanotag solutions were subsequently diluted from $11 \mathrm{pM}$ to $900 \mathrm{fM}$ and SESORS spectra through $5 \mathrm{~mm}$ of tissue were obtained. The solutions were diluted using deionised water. The limit of detection (LOD) was calculated to be 3 times the standard deviation of the blank, divided by the gradient of the straight line of best fit. Error bars represent one standard deviation resulting from 3 replicate samples and 5 scans of each.

\section{Data processing}

All spectra were processed using Matlab software (Version 2017a, The MathWorks, Natrick, MA, USA). Preprocessing involved truncating and baselining the spectra.

\section{Results and Discussion}

To investigate the advantage of using resonant molecules for improved limits of detection of nanotags, gold nanoparticles (AuNPs) were synthesised according to previously reported methods. ${ }^{24}$ The resulting particles had an average diameter of 78 $\mathrm{nm}$. AuNPs were functionalised with either a resonant chalcogenpyrylium Raman reporter, dye 823 , or the commercially available small molecule reporter 1,2-bis(4pyridyl)ethylene, BPE, to create nanotags. Dye 823 is named according to its absorption maximum, i.e. it absorbs at $823 \mathrm{~nm}$, making it resonant with the incident laser light of $830 \mathrm{~nm}$. The resonance capabilities are controlled using previously reported methods. ${ }^{25,24} \mathrm{BPE}$ is a non-resonant small molecule which is often used in SERS applications. It has previously been shown that when a solution of nanotags is obscured by a tissue barrier, superior SERS signal is generated through the use of resonant Raman reporter molecules compared to non-resonant small molecule such as BPE. ${ }^{21}$ In order to demonstrate the advantage of using a resonant Raman reporter molecule over a non-resonant reporter for improved limits of detection (LOD) using SESORS, the ability to detect nanotag solutions containing either BPE or dye 823 at varying concentrations was explored. Solutions of the nanotags were held in a quartz microcuvette and obscured by $5 \mathrm{~mm}$ of porcine tissue and the peak intensity of the most intense peak that corresponded to the reporter molecule in the offset spectra was measured. Significant enhancement in signal was generated through the use of a resonant Raman reporter compared to off resonant molecules. BPE however was shown to offer improved SERS signal through the tissue barrier in comparison to another commercially available non-resonant small molecule, azopyridine (AZPY). ${ }^{21}$ The corresponding SERS spectra and chemical structure of the two Raman reporters are shown in Figure 1, a reference spectrum of the tissue is also displayed. 


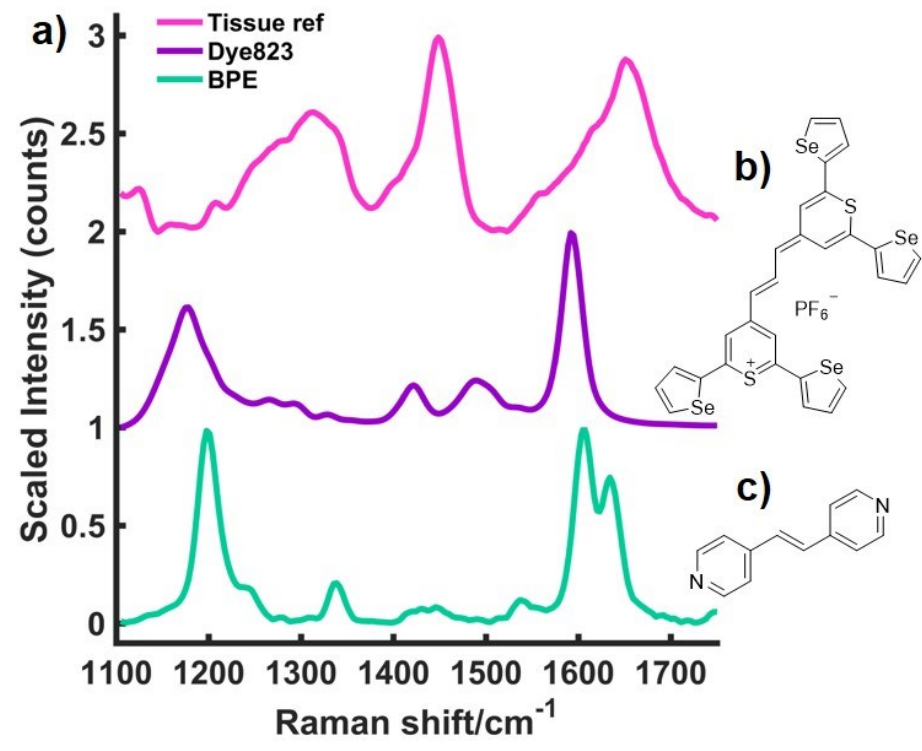

Figure 1 - (a) SERS spectra of the two Raman reporters used in this work, BPE (bottom) and dye 823 (middle), as well as a Raman spectrum of the tissue as a reference (top). Dye 823 is a resonant chalcogen based Raman reporter and BPE is commercially available non-resonant small molecule Raman reporter. Spectra were obtained using the SORS instrument in a conventional Raman mode. All measurements were carried out using a $2 \mathrm{~s}$ integration time, 5 accumulations, $830 \mathrm{~nm}$ laser excitation wavelength. Chemical structure of dye 823 (b) and BPE (c).

Particle dilution studies were conducted in order to calculate a limit of detection for both nanotag solutions through $5 \mathrm{~mm}$ of tissue using SESORS. Each of the two Raman reporters were added to AuNPs to create nanotag solutions with a final dye concentration of $300 \mathrm{nM}$. The aim was to keep the dye concentration as low as possible by exploiting the benefit of using a Raman reporter that is in resonance with the laser. It should be noted that in these studies no inorganic salt was added to the nanotags to enhance the SERS response through the creation of "hot spots". ${ }^{29}$ The concentration of the nanoparticles within the nanotag solution was calculated to be $11.1 \mathrm{pM}$ and subsequent dilutions of the original nanotag suspension, i.e. AuNPs functionalised with either of the two Raman reporters, was carried out using deionised water until no SORS signal from either of the reporter molecules was observed. All measurements were carried out using a handheld SORS instrument using a total exposure time of 10 seconds ( $2 \mathrm{~s}$ integration time, 5 accumulations). The nose cone was fitted to use the instrument in a contact mode setting. The handheld instrument used here has a fixed maximum exposure time, therefore it should be noted that if longer acquisition times were used, the signal to noise ratio may be improved and greater limits of detection could potentially be achieved. To investigate the LOD for each of the two nanotags, solutions with each reporter were held in a quartz microcuvette. Porcine tissue samples of a $5 \mathrm{~mm}$ thickness were placed in front of the cuvette and brought into contact with the laser, leaving no space between the nose cone and the sample (supporting information, Figure S1). The peaks at $1204 \mathrm{~cm}^{-1}$ and $1592 \mathrm{~cm}^{-1}$ were used to calculate the LODs for BPE and dye 823 nanotags respectively since these were the strongest peaks. Spectra were acquired at an $8 \mathrm{~mm}$ spatial offset and truncated and baselined and the peak height at either $1204 \mathrm{~cm}^{-1}$ or $1592 \mathrm{~cm}^{-1}$ was measured at each concentration. We have previously shown that an 8 $\mathrm{mm}$ offset results in the greatest level of through barrier detection. ${ }^{7}$ This is the 
maximum capability of the handheld instrument, i.e. spatial offsets greater than $8 \mathrm{~mm}$ cannot be achieved using this instrument.

The potential of BPE or dye 823 nanotags for use in in vivo applications was then assessed. Figure S2 (supporting information) shows the tracking of BPE nanotags through $5 \mathrm{~mm}$ of tissue at a concentration of $11 \mathrm{pM}$ (middle spectrum) using an $8 \mathrm{~mm}$ offset. The BPE and tissue reference spectra are shown at the top and bottom respectively. The spectral features that correspond to BPE can be clearly seen at this concentration, specifically the peak at $1204 \mathrm{~cm}^{-1}$. However, when the particles were diluted further, i.e. to a concentration of $10 \mathrm{pM}$, they failed to produce a SERS response and the resulting spectra corresponded to that of the tissue alone. Therefore, it was only possible to state an observable LOD of $11 \mathrm{pM}$ through $5 \mathrm{~mm}$ of tissue using BPE nanotags. The sensitivity of BPE nanotags was then compared to nanotags containing dye 823. Figure $2 a$ shows the detection of dye 823 nanotags at a concentration of $6 \mathrm{pM}$ through $5 \mathrm{~mm}$ of tissue using an $8 \mathrm{~mm}$ offset (middle spectrum). The dye reference spectrum is displayed at the top and the tissue reference spectrum at the bottom. Clear detection of the nanotags can be observed at this concentration with almost no spectral contribution from the tissue section observed in the offset spectra, thus demonstrating the efficiency of dye 823 nanotags at a concentration of $6 \mathrm{pM}$. Figure $2 \mathrm{~b}$ shows the particle dilution study of dye 823 nanotag solution through $5 \mathrm{~mm}$ of tissue. The LOD was calculated over a range of 6 to $1 \mathrm{pM}$ using the height of the peak at $1592 \mathrm{~cm}^{-1}$. Figure $2 \mathrm{~b}$ shows that a linear response was obtained and the observable LOD was $1 \mathrm{pM}$. The theoretical LOD was calculated to be $104 \mathrm{fM}$. This was achieved by multiplying the standard deviation of the blank three times and dividing it by the gradient of the straight line, which can be observed in Figure $2 \mathrm{~b}$. Tissue spectra collected at an $8 \mathrm{~mm}$ offset were used as the blank (supporting information, Figure S3). Offset spectra were used as the blank to account for poorer resolution and signal to noise when using spectra collected at an $8 \mathrm{~mm}$ offset. The peak intensity of $1592 \mathrm{~cm}^{-1}$ is also plotted in Figure $2 \mathrm{c}$ and shows the decline in peak intensity as the concentration of the nanotag is reduced. Therefore, it can be stated that the observable limit of detection of nanotags through $5 \mathrm{~mm}$ of tissue using SESORS containing dye 823 is eleven times higher than nanotags containing BPE, i.e. $1 \mathrm{pM}$ (dye 823) compared to $11 \mathrm{pM}$ (BPE). Furthermore, it should be noted that if an additional tissue section was placed behind the cuvette, it is probable that even lower limits of detection would have been achieved since it would help to facilitate the return of scattered Raman photons to the collection optics. 



Figure 2(a) - Dye 823 nanotags at a concentration of 6 pM obscured by $5 \mathrm{~mm}$ of tissue (middle). Dye 823 and tissue reference spectra for the tracking of dye 823 nanotag solution through $5 \mathrm{~mm}$ of tissue. The tissue and dye 823 reference spectra are shown at the bottom and top respectively. The middle spectrum represents the Raman signal collected at an $8 \mathrm{~mm}$ offset through $5 \mathrm{~mm}$ of tissue. Nanotags were obscured by $5 \mathrm{~mm}$ of tissue and held in a quartz microcuvette. (b) SERS particle dilution study for dye 823 nanotags obscured by $5 \mathrm{~mm}$ tissue over the concentration range of $6 \mathrm{pM}$ to $1 \mathrm{pM}$. From the graph, the observed limit of detection of nanotags containing dye 823 is $1 \mathrm{pM}$. The theoretical limit of detection was calculated to be $104 \mathrm{fM}$. The limit of detection was determined by calculating the peak intensity at $1592 \mathrm{~cm}^{-1}$ in the $8 \mathrm{~mm}$ offset spectra. Nanotags were obscured by $5 \mathrm{~mm}$ of tissue and held in a quartz microcuvette. (c) The peak intensity at $1592 \mathrm{~cm}^{-1}$. Peak intensities were obtained by scanning 3 replicate samples, 5 times. The average peak intensity for each of the $\mathbf{5}$ dyes is shown and error bars represent \pm one standard deviation. All measurements were carried out using a $2 \mathrm{~s}$ integration time, 5 accumulations, $830 \mathrm{~nm}$ laser excitation wavelength.

The chalcogen nanotag, which is in resonance with the laser wavelength of $830 \mathrm{~nm}$, produces highly intense SERRS spectra due to its highly aromatic structure. Thus, by making use of the SORS technique, it is possible to obtain a vibrational fingerprint of dye 823 nanotags at low concentrations, even when obscuring tissue barriers are present. Dye 823 also has a larger Raman cross section in comparison to BPE, making it a superior Raman scatterer as well as being in resonance with the excitation wavelength. ${ }^{23}$ Figure $3(\mathrm{a})$ shows the $8 \mathrm{~mm}$ offset spectra of BPE nanotags (green) and dye 823 nanotags (purple) at a concentration of $11 \mathrm{pM}$. As shown, there is a clear difference in the signal to noise ratio between the two nanotags. The resonant Raman reporter produces clearly observable spectra with excellent signal to noise. This is in contrast to the spectra collected of the BPE nanotags at the same concentration through $5 \mathrm{~mm}$ of tissue, in which several spectral features correspond to that of the tissue (supporting information, Figure S3). The relative peak intensities of the most intense peak that corresponds to that of either nanotag, $1592 \mathrm{~cm}^{-1}$ (dye 823) and 1204 $\mathrm{cm}^{-1}$ (BPE), are also shown, Figure 3b. Dye 823 generated the strongest intensity and is therefore assigned an intensity value of $100 \%$. The relative peak intensity refers to the peak intensity of nanotags containing BPE obscured by $5 \mathrm{~mm}$ of tissue, relative to the peak intensity observed using dye 823, expressed as a percentage. As shown, there is almost a $100 \%$ increase in signal when a resonant Raman reporter is used at 
a nanotag concentration of $11 \mathrm{pM}$. Thus, the benefit of red-shifted chalcogenpyrylium Raman reporters for ultra-sensitive SESORS applications, is further demonstrated.

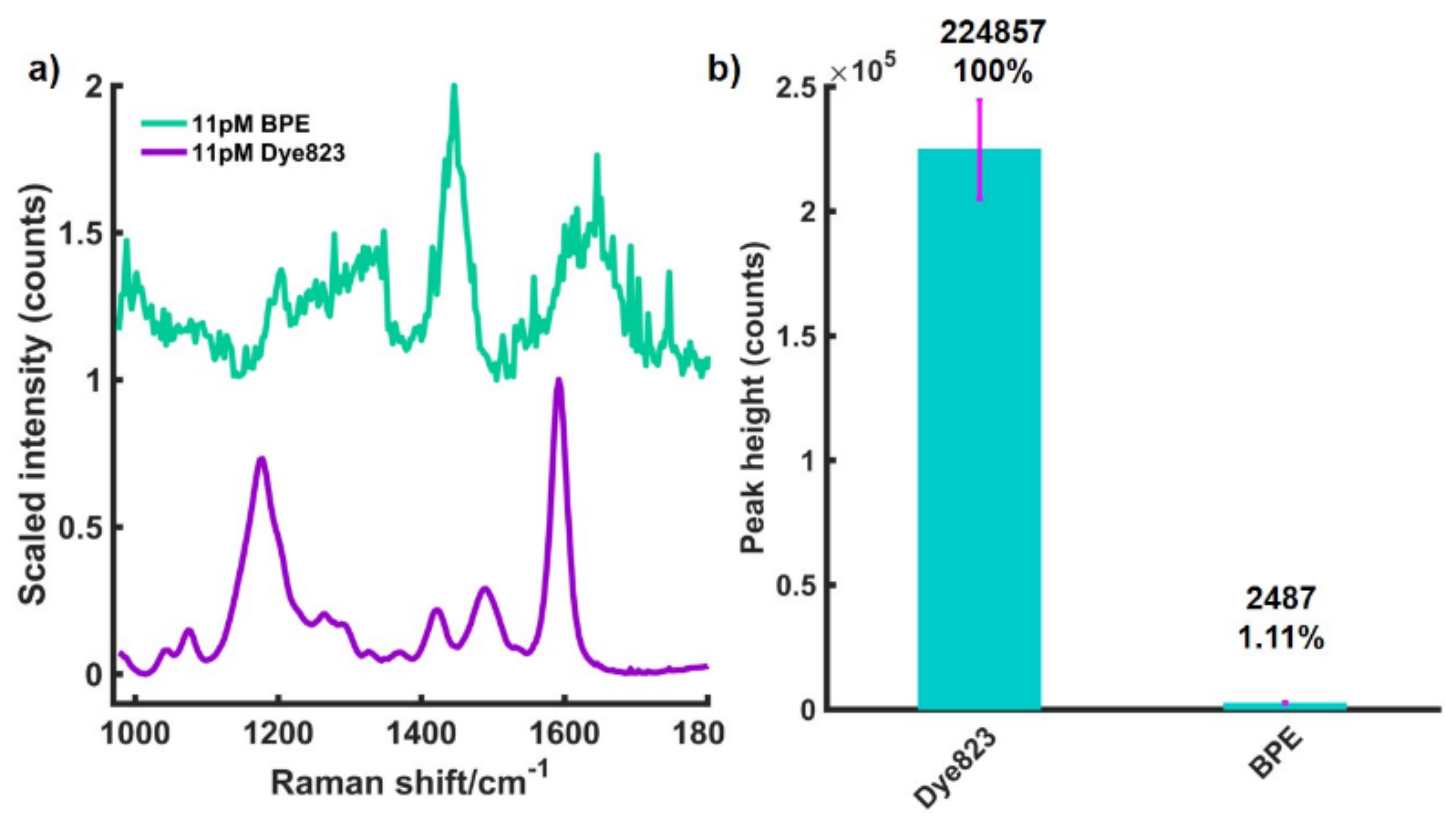

Figure 3 - (a) Scaled $8 \mathrm{~mm}$ offset spectra of 11 pM BPE nanotags (top) and dye 823 nanotags (bottom) obscured by $5 \mathrm{~mm}$ of tissue. (b) Bar chart showing average peak intensities of dye 823 and BPE at 1592 $\mathrm{cm}^{-1}$ and $1204 \mathrm{~cm}^{-1}$ respectively, as well as the relative percentage peak intensity relative to the most intense signal from dye 823, through $5 \mathrm{~mm}$ of tissue. Nanotag solutions were held in a cuvette and the cuvette was placed behind tissue samples. Peak intensities were obtained by scanning 3 replicate samples, 5 times and error bars represent one standard deviation. All measurements were carried out using a $2 \mathrm{~s}$ integration time, 5 accumulations, $830 \mathrm{~nm}$ laser excitation wavelength.

By ultilising a resonant chalcogenpyrylium Raman reporter for SESORRS applications, superior limits of detection can be achieved in contrast to commercially available non-resonant small molecules. The observable limits of detection for nanotags containing dye 823 are eleven times greater than what is seen when nanotags containing BPE are obscured by $5 \mathrm{~mm}$ of tissue, i.e $1 \mathrm{pM}$ in comparison to $11 \mathrm{pM}$ respectively. The work presented here explores the benefit of using resonant Raman reporters for superior low level limits of detection of SERRS nanotags using the SORS technique. It demonstrates the suitability of red-shifted nanotags for biomedical imaging applications where it may be important to keep the nanotag concentration as low as possible. Furthermore, if nanoparticles were used to target a tumour, their accumulation within the tumour would also be low, particularly if they are administered systemically. Therefore, it is important to understand the number of nanoparticles that can be detected at a given depth and also to establish the minimum concentration of nanotags required for SERRS response at a given depth using SORS. In addition, the average thickness of skin on breast tissue ranges from $0.5 \mathrm{~mm}$ to $3 \mathrm{~mm},{ }^{30}$ thus making the detection of SERRS nanotags at low level concentrations through $5 \mathrm{~mm}$ of tissue significant. Future work will focus on establishing the minimum concentration of dye 823 nanotags required to produce a SERRS response at larger, more clinically relevant depths using SORS as well as investigating the use of red shifted nanomaterials for SESORRS applications. 


\section{Acknowledgements}

FN wishes to acknowledge the help of Stuart Bonthron at Cobalt Light Systems, now part of Agilent.

\section{Conflict of Interest}

The authors declare no conflict of interest

\section{Funding}

This work was supported by DSTL and the Engineering and Physical Sciences Research Council [grant numbers EP/J500550/1 and EP/M506643/1, KF and FN and EP/ L014165/1, SM, DG and KF] and by the NSF [grant number CHE-1566142, KP and MRD]. Research data associated with this paper will become available through the following link: http://dx.doi.org/10.15129/d594ff1c-9d11-4e6b-aeea78c8a1864499 


\section{References}

1 P. Matousek, Chem. Soc. Rev., 2007, 36, 1292-1304.

2 P. Matousek, I. P. Clark, E. R. C. Draper, M. D. Morris, a E. Goodship, N.

Everall, M. Towrie, W. F. Finney and A. W. Parker, Appl. Spectrosc., 2005, 59, 393-400.

3 P. Matousek, E. Draper, A. Goodship, I. Clark, K. Ronayne and A. W. Parker, Appl. Spectrosc., 2006, 60, 758-763.

4 C. Eliasson, N. a Macleod and P. Matousek, Anal. Chem., 2007, 79, 8185-9.

5 M. Bloomfield, P. W. Loeffen and P. Matousek, Proc. SPIE, 2010, 7838, 783808-1-783808-15.

6 B. Zachhuber, C. Gasser, E. T. H. Chrysostom and B. Lendl, Anal. Chem., 2011, 83, 9438-9442.

$7 \quad$ F. Nicolson, L. E. Jamieson, S. Mabbott, N. C. Shand, D. Graham and K. Faulds, J. Raman Spectrosc., 2017, 48, 1828-1838.

8 D. I. Ellis, R. Eccles, Y. Xu, J. Griffen, H. Muhamadali, P. Matousek, I. Goodall and R. Goodacre, Sci. Rep., 2017, 7, 12082.

9 K. Buckley, C. G. Atkins, D. Chen, H. G. Schulze, D. V Devine, M. W. Blades and R. F. B. Turner, Analyst, 2016, 141, 1678.

10 K. Sowoidnich, J. H. Churchwell, K. Buckely, A. E. Goodship, A. W. Parker and P. Matousek, Analyst, 2017, 142, 3219.

11 A. Ghita, P. Matousek and N. Stone, J. Biophotonics, , DOI:10.1002/jbio.201600260.

12 R. Goodacre, D. Graham and K. Faulds, TrAC - Trends Anal. Chem., 2018, 102, 359-368.

13 B. Sharma, R. R. Frontiera, A.-I. Henry, E. Ringe and R. P. Van Duyne, Mater. Today, 2012, 15, 16-25.

14 A. Oseledchyk, C. Andreou, M. A. Wall and M. F. Kircher, ACS Nano, 2017, $11,1488$.

15 Y. W. Wang, S. Kang, A. Khan, P. Q. Bao and J. T. C. Liu, Biomed. Opt. Express, 2015, 6, 3714.

16 N. Stone, K. Faulds, D. Graham and P. Matousek, Anal. Chem., 2010, 82, 3969-3973.

17 N. Stone, M. Kerssens, G. R. Lloyd, K. Faulds, D. Graham and P. Matousek, Chem. Sci., 2011, 2, 776-780.

18 K. Ma, J. M. Yuen, N. C. Shah, J. T. Walsh, M. R. Glucksberg and R. P. Van Duyne, Anal Chem, 2011, 83, 9146-9152.

19 B. Sharma, K. Ma, M. R. Glucksberg and R. P. Van Duyne, J. Am. Chem. Soc., 2013, 135, 17290-17923.

20 A. S. Moody, P. C. Baghernejad, K. R. Webb and B. Sharma, Anal. Chem., 2017, 89, 5688-5692.

21 F. Nicolson, L. E. Jamieson, S. Mabbott, K. Plakas, N. Shand, M. Detty, D. Graham and K. Faulds, Chem. Sci., 2018, 9, 3788-3792.

22 F. Nicolson, L. E. Jamieosn, S. Mabbott, K. Plakas, N. C. Shand, D. Graham and K. Faulds, Chem. Commun., 2018, 54, 8530-8533.

23 F. Nicolson, L. E. Jamieson, S. Mabbott, K. Plakas, N. C. Shand, M. R. Detty, D. Graham and K. Faulds, Analyst.

24 M. A. Bedics, H. Kearns, J. M. Cox, S. Mabbott, F. Ali, N. C. Shand, K. Faulds, J. B. Benedict, D. Graham and M. R. Detty, Chem. Sci., 2015, 6, 2302-2306. 
25 H. Kearns, M. A. Bedics, N. C. Shand, K. Faulds, M. R. Detty and D. Graham, Analyst, 2016, 141, 5062-5065.

26 N. C. Shand, SPIE Eur. Secur. Def., 2008, 7119, 71190J-71190J-12.

27 W. Leng, P. Pati and P. J. Vikesland, Environ. Sci. Nano, 2015, 2, 440-453.

28 S. Harmsen, M. A. Bedics, M. A. Wall, R. Huang, M. R. Detty and M. F. Kircher, Nat. Commun., 2015, 6, 6570.

29 H. Kearns, N. C. Shand, W. E. Smith, K. Faulds and D. Graham, Phys. Chem. Chem. Phys., 2015, 17, 1980-6.

30 P. R. Bakic, C. Zhang and A. D. A. Maidment, Med. Phys., 2011, 38, 31653176. 